\section{Research News}

\section{Sporadic cancer model}

Researchers have developed a novel gene-targeting procedure to create mice that more closely recapitulate the spontaneous oncogene activation observed in human cancers. Although transgenic mouse models have been of tremendous use in studying the in vivo mechanisms of oncogenic transformation, this system only allows for transgenes to be expressed in every cell of the animal, or at least a defined anatomical locus. Sporadic cancer, however, is believed to arise from individual cells that succumb to genetic lesions. In the 26 April issue of Nature, Johnson et al. report a new genetic approach to emulate the clonal origin of cancer. They englneered a mouse to express an oncogenic K-ras mutant in a stochastic fashion from the endogenous gene locus by utilizing rare spontaneous recombination events. These mice are highly predisposed to earlyonset lung cancer, and also show increased rates of lymphoma and skin papilloma. Additional deletion of $\mathrm{p} 53$, as seen in many human cancers, accelerates the progression of lung tumors. The complex interactions of transformed cells within their normal microenvironment have a key role in sporadic tumor development. Therefore, this model will more faithfully reflect the oncogenic activation of K-ras in response to environmental factors, such as the chemical carcinogens contained in tobacco smoke.

\section{Natural killer gene}

Cytomegalovirus (CMV) is the leading cause of congenital viral disease and opportunistic infection in immunocompromised patients. In mice, susceptibility to infection by CMV is controlled by one genetic locus, Cmv1, which maps to a cluster of genes encoding natural kdller (NK)-cell receptors. In the May issue of Nature Genetis, Lee et al. show that $\mathrm{Cmv} 1$ is allelic to the stimulatory receptor gene $\mathrm{Klra} 8$ (formerly $\mathrm{Ly} 49 \mathrm{H}$ ). $\mathrm{Klra} 8$ is required in NK cells to allow the detection and destruction of CMV-infected cells. As other loci are involved in natural immunity against other viruses, $K \operatorname{lra} 8$ could have evolved specifically to oppose CMV's evading strategies. NK-cell activity, initially thought to be anti-tumor, had been suggested to contribute to host defense against infections. Lee et al. provide the first direct evidence for a specific role of NK cells in pathogen-specific immune response.

\title{
Spermatogenesis factor
}

The gene $T L F$ (also known as $\operatorname{Trf}$ ), which encodes a close relative of the general transcription factor TATA-binding protein (TBP), has been recently shown to be essential for sperm production in mice. TBP is a central component of the transcription machinery, and by analogy TLF/Trf2 is thought to play a role in the transcription of a specific, as yet undefined, subset of genes. Previous experiments in roundworms, frogs and zebrafish have shown that TLF/Trf2 is required for the

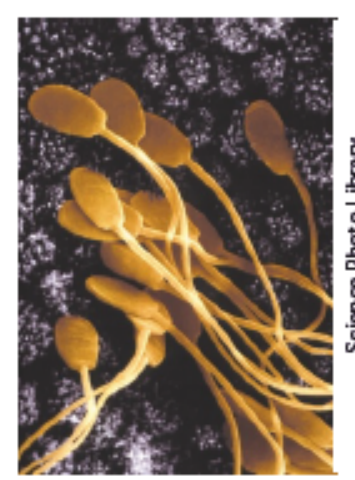

the March issue of Molecular Cell, and Zhang et al. in the 12 April issue of Sclence, report that loss of TLF/Trf2 in the mouse has no effect on viability. In fact, the animals seem normal in nearly all respects, but males are infertile and do not complete the late stages of sperm production. This work suggests that the specific functions of TLF/Trf2 may differ significantly between mammals and other organisms, with TLF/Trf2 taking on a specialized function in marnmalian sperm production. Finally, the findings may have implications for the diagnosis and treatment of male infertility.

development and survival of the embryo, further highlighting the importance of this gene. Now two groups, Martianov et al. in

\section{A new partner for BRCA1}

An investigation into BRCA1 function has lead to the identification of a potential breast cancer gene. Germline mutations in the tumor suppressor gene BRCA1 are known to increase the lifetime risk of developing breast and ovarian cancers, yet almost $90 \%$ of cases cannot be associated with a specific genetic factor. The BRCA1 gene product interacts with a variety of protein partners to mediate a broad range of cellular functions including DNA repair and transcriptional control. In the 6 April issue of Cell, Cantor et al. report that a novel protein interacts directly with BRCA1 and contributes to its DNA repair function. The protein binds to the carboxy terminus of BRCA1, the region that contains most of the diseaseassociated mutations. The authors observed that this protein contained a helicase domain, and therefore named the protein BACH1, for BRCA1-associated $\mathrm{C}$-terminal helicase. Introduction of a missense mutation in BACH1's helicase domain interfered with DNA repair activity in transfected cells, and this interference is dependent on the ability of BACH1 to interact with BRCA1. Furthermore, germline mutations in the BACH1 helicase domain were detected in two early-onset breast cancer patients but not in 200 matched controls. Larger epidemiological studies and the creation of Bach1-null mice will determine whether this gene product has a direct role in cancer suppression.

\section{Retroactive schizophrenia}

The discovery that some people suffering from schizophrenia harbor retroviral RNA sequences suggests that antiviral treatment may someday be used to prevent or treat this neurological disorder. Approximately $1 \%$ of the population develop schizophrenia during their lifetime, experiencing terrfying symptoms such as hearing internal voices and believing that other people are plotting to harm them. In the $10 \mathrm{April}$ issue of PNAS, Karlsson et al. report the identification of retroviral sequences in cerebrospinal fluids of $29 \%$ of people with recent-onset schizophrenia, and $5 \%$ with chronic schizophrenia. The authors were not able to detect retroviral RNA in samples from non-schizophrenic individuals. The greater frequency of retroviral activity in patients with recentonset rather than chronic schizophrenia suggests that activation of retroviral genes may contribute the initial progression of the disease. Reports that schizophrenics suffer a fever immediately before the onset of symptorns support this theory. The human genome harbors many endogenous retroviral genes, and retroviruses have been previously associated with schizophrenia, as well as a number of other diseases including multiple sclerosis and diabetes. Schizophrenia also has a genetic and errvironmental basis, and it is possible that retroviruses only cause disease in people that are predisposed to retroviral activation. There are some treatments for disease symptoms, but most people with schizophrenia continue to suffer some symptoms throughout their lives. It has been estimated that no more than one in five individuals completely recovers, but these findings may lead to new therepeutics.

By Leila Alland, Tanguy Chouard, Kristine Novak, Bernd Pulverer \& Charlotte Wang 